\title{
Two-Dimensional Direction of Arrival (DOA) Estimation for Rectangular Array via Compressive Sensing Trilinear Model
}

\author{
Huaxin Yu, ${ }^{1}$ Xiaofeng Qiu, ${ }^{2}$ Xiaofei Zhang, ${ }^{1,3}$ Chenghua Wang, ${ }^{1}$ and Gang Yang ${ }^{1}$ \\ ${ }^{1}$ Key Laboratory of Radar Imaging and Microwave Photonics, Nanjing University of Aeronautics and Astronautics, \\ Ministry of Education, Nanjing 210016, China \\ ${ }^{2}$ Institute of Command Information System, PLA University of Science and Technology, Nanjing 210007, China \\ ${ }^{3}$ Laboratory of Modern Acoustics of Ministry of Education, Nanjing University, Nanjing 210093, China
}

Correspondence should be addressed to Xiaofei Zhang; njxnd88@126.com

Received 15 March 2014; Revised 21 July 2014; Accepted 17 September 2014

Academic Editor: Hang Hu

Copyright (C) 2015 Huaxin Yu et al. This is an open access article distributed under the Creative Commons Attribution License, which permits unrestricted use, distribution, and reproduction in any medium, provided the original work is properly cited.

We investigate the topic of two-dimensional direction of arrival (2D-DOA) estimation for rectangular array. This paper links angle estimation problem to compressive sensing trilinear model and derives a compressive sensing trilinear model-based angle estimation algorithm which can obtain the paired 2D-DOA estimation. The proposed algorithm not only requires no spectral peak searching but also has better angle estimation performance than estimation of signal parameters via rotational invariance techniques (ESPRIT) algorithm. Furthermore, the proposed algorithm has close angle estimation performance to trilinear decomposition. The proposed algorithm can be regarded as a combination of trilinear model and compressive sensing theory, and it brings much lower computational complexity and much smaller demand for storage capacity. Numerical simulations present the effectiveness of our approach.

\section{Introduction}

Array signal processing has received a significant amount of attention during the last decades due to its wide application in radar, sonar, radio astronomy, and satellite communication [1]. The direction of arrival (DOA) estimation of signals impinging on an antenna array is a fundamental problem in array signal processing, and many DOA estimation methods [2-7] have been proposed for its solution. They contain estimation of signal parameters via rotational invariance techniques (ESPRIT) algorithm [2,3], multiple signal classification (MUSIC) algorithm [4], Root-MUSIC [5], matrix pencil methods [6], and so on. Compared with linear arrays, uniform rectangular array can identify twodimensional DOA (2D-DOA). 2D-DOA estimation with rectangular array has received considerable attention in the field of array signal processing [7-12]. ESPRIT algorithms in [8-11] have exploited the invariance property for 2DDOA estimation in uniform rectangular array. Parallel factor analysis (PARAFAC) in [12], which is also called trilinear decomposition method, was proposed for 2D-DOA estimation for uniform rectangular array, and it has better angle estimation performance than ESPRIT. MUSIC algorithm, as a subspace method, has good angle estimation performance and matches irregular arrays. It has been proved that twodimensional MUSIC (2D-MUSIC) algorithm [13] can be used for 2D-DOA estimation. However, the requirement of twodimensional (2D) spectrum searching renders much higher computational complexity.

Compressive sensing $[14,15]$ has attracted a lot of attention recently, and it has been applied to image processing, machine learning, channel estimation, radar imaging, and penalized regression [16]. According to the theory of compressive sensing, a signal that is sparse in some domain can be recovered via fewer samples than required by the Nyquist sampling theorem. The DOAs of sources form a sparse vector in the potential signal space, and, therefore, compressive sensing can be applied to DOA estimation. 
The superresolution property and ability of resolving coherent sources can be achieved when we apply it to the source location [17]. Lots of the DOA estimation methods with compressive sensing just use one snapshot and are very sensitive to the noise. For multiple snapshots, $\ell_{1}$-SVD method [16] employed $\ell_{1}$ norm to enforce sparsity and singular value decomposition to reduce complexity and sensitivity to noise, and sparse recovery for weighted subspace fitting in [17] improved the $\ell_{1}$-SVD method via the weight to the subspace.

Compared to matrix decomposition, trilinear decomposition has a distinctive and attractive feature: it is often unique [18-22]. In the signal processing field, trilinear decomposition can be regarded as a generalization of ESPRIT and joint approximate diagonalization [19-22]. The compressive sensing trilinear model-based algorithm discussed in this paper can be regarded as a combination of trilinear model and compressive sensing theory, which brings much lower computational complexity and much smaller demand for storage capacity.

The framework of compressive sensing for sparse lowrank tensor is proposed in [23] and used for signal detection and multiple-input-multiple-output radar in $[24,25]$. In this paper, the problem of $2 \mathrm{D}-\mathrm{DOA}$ estimation for rectangular array is linked to compressive sensing trilinear model. Exploiting this link, we derive a compressive sensing trilinear model-based 2D-DOA estimation algorithm for rectangular array. Firstly, we compress the received data to get a compressed trilinear model and then obtain the estimates of compressed direction matrices through performing trilinear decomposition for the compressed model. Finally, we formulate a sparse recovery problem through the estimated compressed direction matrices and apply the orthogonal matching pursuit (OMP) [26] to resolve it for 2D-DOA estimation. Due to compression, the proposed method has much lower computational complexity than conventional trilinear decomposition method [12] and 2D-MUSIC algorithm and requires much smaller storage capacity. We illustrate that the proposed algorithm has better angle estimation performance than ESPRIT algorithm. Furthermore, our algorithm can obtain paired elevation angles and azimuth angles automatically. We also derive the Cramer-Rao bound (CRB) for 2DDOA estimation in rectangular array. Numerical simulations present the effectiveness of our approach.

The remainder of this paper is structured as follows. Section 2 presents the data model, and Section 3 proposes the compressed sensing trilinear model-based algorithm for $2 \mathrm{D}-\mathrm{DOA}$ estimation in rectangular array. In Section 4 , the simulation results are presented to verify improvement of the proposed algorithm, while the conclusions are drawn in Section 5.

Notation. Bold lower (upper) case letters are adopted to represent vectors (matrices). $(\cdot)^{T},(\cdot)^{H},(\cdot)^{*},(\cdot)^{-1}$, and $(\cdot)^{+}$denote transpose, conjugate transpose, conjugate, matrix inversion, and pseudoinverse operations, respectively. $\mathbf{I}_{P}$ stands for a $P \times P$ identity matrix. $D_{n}(\mathbf{A})$ denotes a diagonal matrix with the entries of the matrix A's $n$th row on the main diagonal. The $i$ th entry of a given column vector $\mathbf{g}$ is denoted by $\mathbf{g}(i)$. $\odot, \otimes$, and $\oplus$ denote Khatri-Rao product, Kronecker product, and Hadamard product, respectively.

If $\mathbf{A}$ is a $p$-by- $q$ matrix and $\mathbf{B}$ is an $m$-by- $n$ matrix, then the Kronecker product $\mathbf{A} \otimes \mathbf{B}$ is the $m p$-by-nq block matrix:

$$
\mathbf{A} \otimes \mathbf{B}=\left[\begin{array}{cccc}
a_{11} \mathbf{B} & a_{12} \mathbf{B} & \cdots & a_{1 q} \mathbf{B} \\
a_{21} \mathbf{B} & a_{22} \mathbf{B} & \cdots & a_{2 q} \mathbf{B} \\
\vdots & \vdots & \ddots & \vdots \\
a_{p 1} \mathbf{B} & a_{p 2} \mathbf{B} & \cdots & a_{p q} \mathbf{B}
\end{array}\right]
$$

where $a_{i j}$ is the $(i, j)$ element of the matrix $\mathbf{A}$.

If $\mathbf{A}$ is an $I$-by- $F$ matrix and $\mathbf{B}$ is a $J$-by- $F$ matrix, then the Khatri-Rao product $\mathbf{A} \odot \mathbf{B}$ is the $I J$-by- $F$ block matrix:

$$
\mathbf{A} \odot \mathbf{B}=\left[\mathbf{a}_{1} \otimes \mathbf{b}_{1}, \ldots, \mathbf{a}_{F} \otimes \mathbf{b}_{F}\right],
$$

where $\mathbf{a}_{f}$ and $\mathbf{b}_{f}$ are the $f$ th column of the matrices $\mathbf{A}$ and $\mathbf{B}$, respectively.

If $\mathbf{A}$ is an $I$-by- $J$ matrix and $\mathbf{B}$ is an $I$-by- $J$ matrix, then the Hadamard product $\mathbf{A} \oplus \mathbf{B}$ is

$$
\mathbf{A} \oplus \mathbf{B}=\left[\begin{array}{cccc}
a_{11} b_{11} & a_{12} b_{12} & \cdots & a_{1 J} b_{1 J} \\
a_{21} b_{21} & a_{22} b_{22} & \cdots & a_{2 J} b_{2 J} \\
\vdots & \vdots & \ddots & \vdots \\
a_{I 1} b_{I 1} & a_{I 2} b_{I 2} & \cdots & a_{I J} b_{I J}
\end{array}\right]
$$

where $a_{i j}$ and $b_{i j}$ are the $(i, j)$ element of the matrices $\mathbf{A}$ and $\mathbf{B}$, respectively.

\section{Data Model}

A rectangular array consisted of $M \times N$ elements is shown in Figure 1, where the distance between two adjacent elements is $d$. We consider signals in the far field, in which case the signal sources are far away enough that the arriving waves are essentially planes over the array. We assume that the noise is independent of the sources. It is also assumed that there are $K$ noncoherent or independent sources, and the number of sources is preknown. $\theta_{k}$ and $\phi_{k}$ are the elevation angle and the azimuth angle of the $k$ th source, respectively. We assume the sources impinge on the array with different DOAs.

The received signal of the first subarray in the rectangular array is $\mathbf{x}_{1}(t)=\mathbf{A}_{x} \mathbf{s}(t)+\mathbf{n}_{1}(t)$, where $\mathbf{A}_{x}=\left[\mathbf{a}_{x}\left(\phi_{1}, \theta_{1}\right)\right.$, $\left.\mathbf{a}_{x}\left(\phi_{2}, \theta_{2}\right), \ldots, \mathbf{a}_{x}\left(\phi_{K}, \theta_{K}\right)\right] \in \mathbf{C}^{M \times K}$ with $\mathbf{a}_{x}\left(\phi_{k}, \theta_{k}\right)=[1$, $\left.e^{j 2 \pi d \sin \theta_{k} \cos \phi_{k} / \lambda}, \ldots, e^{j 2 \pi(M-1) d \sin \theta_{k} \cos \phi_{k} / \lambda}\right]^{T}$ and $\lambda$ is the wavelength. $\mathbf{n}_{1}(t)$ is the received additive white Gaussian noise of the first subarray. $\mathbf{s}(t) \in \mathbf{C}^{K \times 1}$ is the source vector. The received signal of the $n$th subarray in the rectangular array is $\mathbf{x}_{n}(t)=\mathbf{A}_{x} \Phi^{n-1} \mathbf{s}(t)+\mathbf{n}_{n}(t)$, where $\Phi=$ $\operatorname{diag}\left(e^{j 2 \pi d \sin \theta_{1} \sin \phi_{1} / \lambda}, \ldots, e^{j 2 \pi d \sin \theta_{K} \sin \phi_{K} / \lambda}\right)$ and $\mathbf{n}_{n}(t)$ is the received additive white Gaussian noise of the $n$th subarray. Therefore, the received signal of the rectangular array is [27]

$$
\mathbf{x}(t)=\left[\begin{array}{c}
\mathbf{x}_{1}(t) \\
\mathbf{x}_{2}(t) \\
\vdots \\
\mathbf{x}_{N}(t)
\end{array}\right]=\left[\begin{array}{c}
\mathbf{A}_{x} \\
\mathbf{A}_{x} \Phi \\
\vdots \\
\mathbf{A}_{x} \Phi^{N-1}
\end{array}\right] \mathbf{s}(t)+\left[\begin{array}{c}
\mathbf{n}_{1}(t) \\
\mathbf{n}_{2}(t) \\
\vdots \\
\mathbf{n}_{N}(t)
\end{array}\right] .
$$




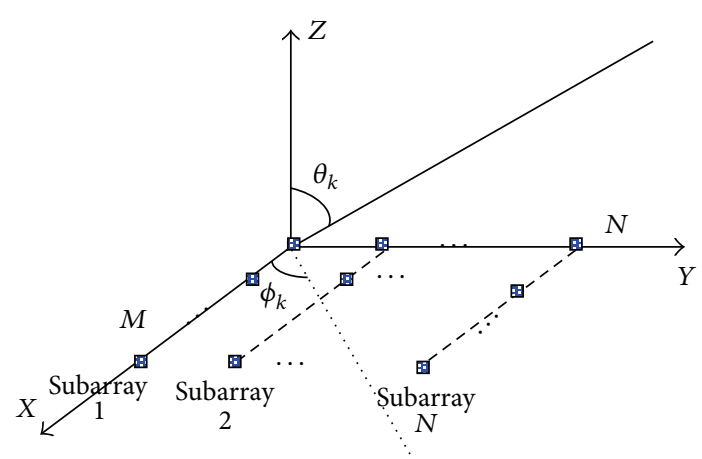

FIGURE 1: The structure of uniform rectangular array.

The signal $\mathbf{x}(t) \in \mathbf{C}^{M N \times 1}$ in (4) can also be denoted by

$$
\mathbf{x}(t)=\left[\mathbf{A}_{y} \odot \mathbf{A}_{x}\right] \mathbf{s}(t)+\mathbf{n}(t),
$$

where $\mathbf{A}_{y}=\left[\mathbf{a}_{y}\left(\phi_{1}, \theta_{1}\right), \mathbf{a}_{y}\left(\phi_{2}, \theta_{2}\right), \ldots, \mathbf{a}_{y}\left(\phi_{K}, \theta_{K}\right)\right]$ with $\mathbf{a}_{y}\left(\phi_{k}, \theta_{k}\right)=\left[1, e^{j 2 \pi d \sin \theta_{k} \sin \phi_{k} / \lambda}, \ldots, e^{j 2 \pi(N-1) d \sin \theta_{k} \sin \phi_{k} / \lambda}\right]^{T} ;$ $\mathbf{n}(t)=\left[\mathbf{n}_{1}(t)^{T}, \mathbf{n}_{2}(t)^{T}, \ldots, \mathbf{n}_{N}(t)^{T}\right]^{T} \in \mathbf{C}^{M N \times 1} . \odot$ denotes Khatri-Rao product.

According to the definition of Khatri-Rao product, the signal in (5) can be rewritten as

$$
\begin{aligned}
& \mathbf{x}(t)=\left[\mathbf{a}_{y}\left(\phi_{1}, \theta_{1}\right) \otimes \mathbf{a}_{x}\left(\phi_{1}, \theta_{1}\right), \ldots,\right. \\
&\left.\mathbf{a}_{y}\left(\phi_{K}, \theta_{K}\right) \otimes \mathbf{a}_{x}\left(\phi_{K}, \theta_{K}\right)\right] \mathbf{s}(t)+\mathbf{n}(t),
\end{aligned}
$$

where $\otimes$ denotes Kronecker product. We collect $L$ samples and define $\mathbf{X}=[\mathbf{x}(1), \mathbf{x}(2), \ldots, \mathbf{x}(L)] \in \mathbf{C}^{M N \times L}$, which can be expressed as

$$
\begin{aligned}
& \mathbf{X}=\left[\mathbf{A}_{y} \odot \mathbf{A}_{x}\right] \mathbf{S}^{T}+\mathbf{N}=\left[\begin{array}{c}
\mathbf{X}_{1} \\
\mathbf{X}_{2} \\
\vdots \\
\mathbf{X}_{N}
\end{array}\right] \\
& =\left[\begin{array}{c}
\mathbf{A}_{x} D_{1}\left(\mathbf{A}_{y}\right) \\
\mathbf{A}_{x} D_{2}\left(\mathbf{A}_{y}\right) \\
\vdots \\
\mathbf{A}_{x} D_{N}\left(\mathbf{A}_{y}\right)
\end{array}\right] \mathbf{S}^{T}+\left[\begin{array}{c}
\mathbf{N}_{1} \\
\mathbf{N}_{2} \\
\vdots \\
\mathbf{N}_{N}
\end{array}\right],
\end{aligned}
$$

where $\mathbf{S}=[\mathbf{s}(1), \mathbf{s}(2), \ldots, \mathbf{s}(L)]^{T} \in \mathbf{C}^{L \times K}$ is source matrix and $\mathbf{N}=[\mathbf{n}(1), \mathbf{n}(2), \ldots, \mathbf{n}(L)]$ is the received additive white Gaussian noise matrix. $\mathbf{N}_{n} \in \mathbf{C}^{M \times L}(n=1, \ldots, N)$ is the noise matrix. Thus, $\mathbf{X}_{n} \in \mathbf{C}^{M \times L}$ in (7) is denoted as

$$
\mathbf{X}_{n}=\mathbf{A}_{x} D_{n}\left(\mathbf{A}_{y}\right) \mathbf{S}^{T}+\mathbf{N}_{n}, \quad n=1,2, \ldots, N
$$

Equation (7) can also be denoted with the trilinear model [18, 28]

$$
\begin{aligned}
& x_{m, n, l}=\sum_{k=1}^{K} \mathbf{A}_{x}(m, k) \mathbf{A}_{y}(n, k) \mathbf{S}(l, k)+n_{m, n, l}, \\
& m=1, \ldots, M, \quad n=1, \ldots, N, \quad l=1, \ldots, L,
\end{aligned}
$$

where $\mathbf{A}_{x}(m, k)$ is the $(m, k)$ element of the matrix $\mathbf{A}_{x}$ and similarly for the others. $n_{m, n, l}$ is noise part. $\mathbf{X}_{n} \in \mathbf{C}^{M \times L}(n=$ $1, \ldots, N)$ can be regarded as slicing the three-dimensional data in a series of slices, which is shown in Figure 2. There are two more matrix system rearrangements, in which we have $\mathbf{Y}_{m}=\mathbf{S} D_{m}\left(\mathbf{A}_{x}\right) \mathbf{A}_{y}^{T}+\mathbf{N}_{m}^{y}, m=1, \ldots, M$, and $\mathbf{Z}_{l}=$ $\mathbf{A}_{y} D_{l}(\mathbf{S}) \mathbf{A}_{x}^{T}+\mathbf{N}_{l}^{z}, l=1, \ldots, L$, where $\mathbf{N}_{m}^{y}$ and $\mathbf{N}_{l}^{z}$ are noise matrices. Then, we form the matrices of $\mathbf{Y} \in \mathbf{C}^{M L \times N}$ and $\mathbf{Z} \in \mathbf{C}^{L N \times M}$ :

$$
\mathbf{Y}=\left[\begin{array}{c}
\mathbf{Y}_{1} \\
\mathbf{Y}_{2} \\
\vdots \\
\mathbf{Y}_{M}
\end{array}\right]=\left[\mathbf{A}_{x} \odot \mathbf{S}\right] \mathbf{A}_{y}^{T}+\mathbf{N}^{y}
$$

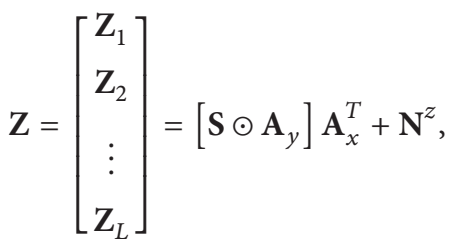

where

$$
\begin{aligned}
& \mathbf{N}^{y}=\left[\begin{array}{c}
\mathbf{N}_{1}^{y} \\
\mathbf{N}_{2}^{y} \\
\vdots \\
\mathbf{N}_{M}^{y}
\end{array}\right], \\
& \mathbf{N}^{z}=\left[\begin{array}{c}
\mathbf{N}_{1}^{z} \\
\mathbf{N}_{2}^{z} \\
\vdots \\
\mathbf{N}_{L}^{z}
\end{array}\right] .
\end{aligned}
$$

\section{2D-DOA Estimation Based on Compressive Sensing Trilinear Model}

We link the problem of 2D-DOA estimation for rectangular array to compressive sensing trilinear model and derive a compressive sensing trilinear model-based 2D-DOA estimation algorithm. Firstly, we compress the received data to get a compressed trilinear model and then obtain the estimates of compressed direction matrices through performing trilinear decomposition for the compressed model. Finally, we formulate the sparse recovery problem for $2 \mathrm{D}$-DOA estimation.

3.1. Trilinear Model Compression. We compress the threeway data $\underline{\mathbf{X}} \in \mathbf{C}^{M \times N \times L}$ into a smaller three-way data $\underline{\mathbf{X}}^{\prime} \in$ $\mathrm{C}^{M^{\prime} \times N^{\prime} \times L^{\prime}}$, where $M^{\prime}<M, N^{\prime}<N$, and $L^{\prime}<L$. The trilinear model compression processing is shown in Figure 3. We define the compression matrices as $\mathbf{U} \in \mathbf{C}^{M \times M^{\prime}}, \mathbf{V} \in \mathbf{C}^{N \times N^{\prime}}$, and $\mathbf{W} \in \mathbf{C}^{L \times L^{\prime}}$, and the compression matrices $\mathbf{U}, \mathbf{V}$, and $\mathbf{W}$ can be generated randomly or obtained by Tucker3 decomposition $[23,29]$. We can use the Tucker3 decomposition, 


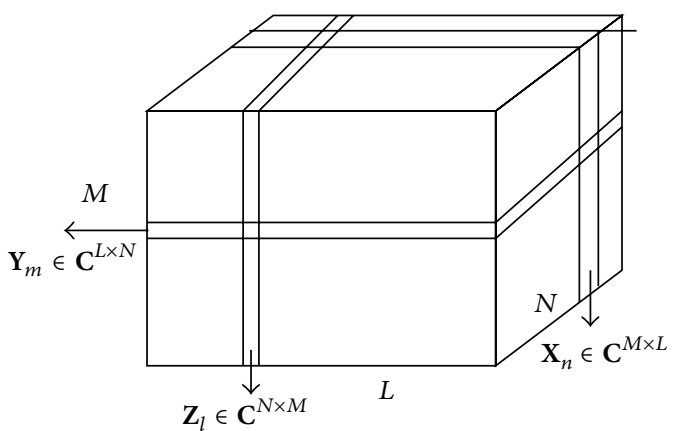

Figure 2: Trilinear model.

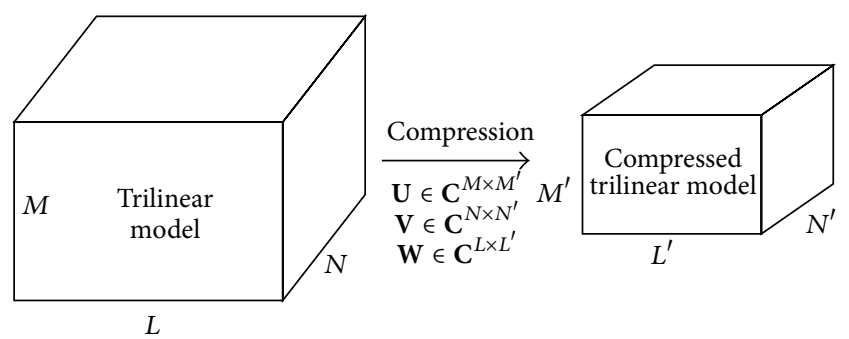

FIGURE 3: The compression of trilinear model.

where tensor is decomposed into the core tensor, to obtain the compression matrices. The compression matrices should satisfy the restricted isometry property. And random Gaussian, Bernoulli, and partial Fourier matrices satisfy the restricted isometry property with number of measurements nearly linear in the sparsity level [30,31]. $\mathrm{C}^{M^{\prime} N^{\prime} \times L^{\prime}}$

Then compress $\mathbf{X} \in \mathbf{C}^{M N \times L}$ in (7) to a smaller one as $\mathbf{X}^{\prime} \epsilon$

$$
\begin{aligned}
\mathbf{X}^{\prime}= & \left(\mathbf{V}^{T} \otimes \mathbf{U}^{T}\right) \mathbf{X} \mathbf{W}^{T} \\
= & \left(\mathbf{V}^{T} \otimes \mathbf{U}^{T}\right)\left[\mathbf{A}_{y} \odot \mathbf{A}_{x}\right] \mathbf{S}^{T} \mathbf{W}^{T} \\
& +\left(\mathbf{V}^{T} \otimes \mathbf{U}^{T}\right) \mathbf{N} \mathbf{W}^{T} .
\end{aligned}
$$

According to the property of Khatri-Rao product [23], we know

$$
\left(\mathbf{V}^{T} \otimes \mathbf{U}^{T}\right)\left[\mathbf{A}_{y} \odot \mathbf{A}_{x}\right]=\left(\mathbf{V}^{T} \mathbf{A}_{y}\right) \odot\left(\mathbf{U}^{T} \mathbf{A}_{x}\right) .
$$

Define $\mathbf{A}_{x}^{\prime}=\mathbf{U}^{T} \mathbf{A}_{x}, \mathbf{A}_{y}^{\prime}=\mathbf{V}^{T} \mathbf{A}_{y}$, and $\mathbf{S}^{\prime}=\mathbf{W}^{T} \mathbf{S}$. Equation (11) is also denoted as

$$
\mathbf{X}^{\prime}=\left[\mathbf{A}_{y}^{\prime} \odot \mathbf{A}_{x}^{\prime}\right] \mathbf{S}^{\prime T}+\mathbf{N}^{\prime},
$$

where $\mathbf{N}^{\prime}=\left(\mathbf{V}^{T} \otimes \mathbf{U}^{T}\right) \mathbf{N} \mathbf{W}^{T} . \mathbf{X}^{\prime}$ can be denoted by trilinear model. With respect to (10) and (11), we form the matrices of $\mathbf{Y}^{\prime}$ and $\mathbf{Z}^{\prime}$ according to the compressed data

$$
\begin{aligned}
& \mathbf{Y}^{\prime}=\left[\mathbf{A}_{x}^{\prime} \odot \mathbf{S}^{\prime}\right] \mathbf{A}_{y}^{\prime T}+\mathbf{N}^{\prime y}, \\
& \mathbf{Z}^{\prime}=\left[\mathbf{S}^{\prime} \odot \mathbf{A}_{y}^{\prime}\right] \mathbf{A}_{x}^{\prime T}+\mathbf{N}^{\prime z},
\end{aligned}
$$

where $\mathbf{N}^{\prime y}$ and $\mathbf{N}^{\prime z}$ are the noise part. The compressed trilinear model may degrade the angle estimation performance.

By trilinear model compression, the proposed method has much lower computational complexity than conventional trilinear decomposition method and requires much smaller storage capacity. Conventional compressive sensing is to compress the matrix, while our algorithm compresses the three-dimensional tensor.

3.2. Trilinear Decomposition. Trilinear alternating least square (TALS) algorithm is an iterative method for estimating the parameters of a trilinear decomposition $[18,28]$. We concisely show the basic idea of TALS: (1) update one matrix each time via LS, which is conditioned on previously obtained estimates of the remaining matrices; (2) proceed to update the other matrices; (3) repeat until convergence of the LS cost function [21, 22]. TALS algorithm is discussed as follows.

According to (15), least squares (LS) fitting is

$$
\min _{\mathbf{A}_{x}^{\prime}, \mathbf{A}_{y}^{\prime}, \mathbf{S}^{\prime}}\left\|\mathbf{X}^{\prime}-\left[\mathbf{A}_{y}^{\prime} \odot \mathbf{A}_{x}^{\prime}\right] \mathbf{S}^{\prime T}\right\|_{F}
$$

and LS update for the matrix $\mathbf{S}^{\prime}$ is

$$
\widehat{\mathbf{S}}^{\prime T}=\left[\widehat{\mathbf{A}}_{y}^{\prime} \odot \widehat{\mathbf{A}}_{x}^{\prime}\right]^{+} \mathbf{X}^{\prime},
$$

where $\widehat{\mathbf{A}}_{x}^{\prime}$ and $\widehat{\mathbf{A}}_{y}^{\prime}$ are previously obtained estimates of $\mathbf{A}_{x}^{\prime}$ and $\mathbf{A}_{y}^{\prime}$, respectively. According to (16), LS fitting is

$$
\min _{\mathbf{A}_{x}^{\prime}, \mathbf{A}_{y}^{\prime}, \mathbf{S}^{\prime}}\left\|\mathbf{Y}^{\prime}-\left[\mathbf{A}_{x}^{\prime} \odot \mathbf{S}^{\prime}\right] \mathbf{A}_{y}^{\prime T}\right\|_{F}
$$

and LS update for $\mathbf{A}_{y}^{\prime}$ is

$$
\widehat{\mathbf{A}}_{y}^{\prime T}=\left[\widehat{\mathbf{A}}_{x}^{\prime} \odot \widehat{\mathbf{S}}^{\prime}\right]^{+} \mathbf{Y}^{\prime},
$$

where $\widehat{\mathbf{A}}_{x}^{\prime}$ and $\widehat{\mathbf{S}}^{\prime}$ stand for the previously obtained estimates of $\mathbf{A}_{x}^{\prime}$ and $\mathbf{S}^{\prime}$. Similarly, according to (17), LS fitting is

$$
\min _{\mathbf{A}_{x}^{\prime}, \mathbf{A}_{y}^{\prime}, \mathbf{S}^{\prime}}\left\|\mathbf{Z}^{\prime}-\left[\mathbf{S}^{\prime} \odot \mathbf{A}_{y}^{\prime}\right] \mathbf{A}_{x}^{\prime T}\right\|_{F},
$$

where $\widetilde{\mathbf{Z}}^{\prime}$ is the noisy compressed signal. LS update for $\mathbf{A}_{x}^{\prime}$ is

$$
\widehat{\mathbf{A}}_{x}^{\prime T}=\left[\widehat{\mathbf{S}}^{\prime} \odot \widehat{\mathbf{A}}_{y}^{\prime}\right]^{+} \mathbf{Z}^{\prime},
$$

where $\widehat{\mathbf{A}}_{y}^{\prime}$ and $\widehat{\mathbf{S}}^{\prime}$ stand for the previously obtained estimates of $\mathbf{A}_{y}^{\prime}$ and $\mathbf{S}^{\prime}$, respectively.

Define $\mathbf{E}=\mathbf{X}-\left[\widehat{\mathbf{A}}_{y}^{\prime} \odot \widehat{\mathbf{A}}_{x}^{\prime}\right] \widehat{\mathbf{S}}^{\prime T}$, where $\widehat{\mathbf{S}}^{\prime}, \widehat{\mathbf{A}}_{y}^{\prime}$, and $\widehat{\mathbf{A}}_{x}^{\prime}$ present the estimates of $\mathbf{S}^{\prime}, \mathbf{A}_{y}^{\prime}$, and $\mathbf{A}_{x}^{\prime}$, respectively. The sum of squared residuals (SSR) in trilinear model is defined as SSR $=\sum_{j=1}^{L} \sum_{i=1}^{M N}\left|e_{i j}\right|$, where $e_{i j}$ is the $(i, j)$ element of the matrix E. According to (19), (21), and (23), the matrices $\mathbf{S}^{\prime}$, $\mathbf{A}_{y}^{\prime}$, and $\mathbf{A}_{x}^{\prime}$ are updated with least squares repeatedly until SSR attain apriorthreshold. The we obtain the final estimates $\widehat{\mathbf{S}}^{\prime}, \widehat{\mathbf{A}}_{y}^{\prime}$, and $\widehat{\mathbf{A}}_{x}^{\prime}$. 
Theorem 1 (see [22]). Considering $\mathbf{X}_{n}^{\prime}=\mathbf{A}_{x}^{\prime} D_{n}\left(\mathbf{A}_{y}^{\prime}\right) \mathbf{S}^{\prime T}, n=$ $1, \ldots, N^{\prime}$, where $\mathbf{A}_{x}^{\prime} \in \mathbf{C}^{M^{\prime} \times K}, \mathbf{S}^{\prime} \in \mathbf{C}^{L^{\prime} \times K}$, and $\mathbf{A}_{y}^{\prime} \in \mathbf{C}^{N^{\prime} \times K}$, if

$$
k_{\mathbf{A}_{x}^{\prime}}+k_{\mathbf{A}_{y}^{\prime}}+k_{\mathbf{S}^{\prime T}} \geq 2 K+2
$$

where $k_{\mathbf{A}}$ is the $k$-rank of the matrix $\mathbf{A}[18]$, then $\mathbf{A}_{x}^{\prime}, \mathbf{S}^{\prime}$, and $\mathbf{A}_{y}^{\prime}$ are unique up to permutation and scaling of columns.

For the different DOAs and independent sources, we have $k_{\mathbf{A}_{x}^{\prime}}=\min \left(M^{\prime}, K\right), k_{\mathbf{A}_{y}^{\prime}}=\min \left(N^{\prime}, K\right)$, and $k_{\mathbf{S}^{\prime T}}=\min \left(L^{\prime}, K\right)$ in the trilinear model in this paper, and then the inequality in (24) becomes

$$
\begin{aligned}
& \min \left(M^{\prime}, K\right)+\min \left(N^{\prime}, K\right)+\min \left(L^{\prime}, K\right) \\
& \quad \geq 2 K+2 .
\end{aligned}
$$

When $M^{\prime} \geq K, N^{\prime} \geq K$, and $L^{\prime} \geq K$, the identifiable condition is $1 \leq K \leq \min \left(M^{\prime}, N^{\prime}\right)$.

When $M^{\prime} \leq K, N^{\prime} \leq K$, and $L^{\prime} \geq K$, the identifiable condition is $\max \left(M^{\prime}, N^{\prime}\right) \leq K \leq M^{\prime}+N^{\prime}-2$. Hence, the proposed algorithm is effective when $K \leq M^{\prime}+N^{\prime}-2$ and the maximum number of sources that can be identified is $M^{\prime}+$ $N^{\prime}-2$.

After the trilinear decomposition, we obtain the estimates of the loading matrices

$$
\begin{gathered}
\widehat{\mathbf{A}}_{x}^{\prime}=\mathbf{A}_{x}^{\prime} \Pi \Delta_{1}+\mathbf{E}_{1}, \\
\widehat{\mathbf{S}}^{\prime}=\mathbf{S}^{\prime} \Pi \Delta_{2}+\mathbf{E}_{2}, \\
\widehat{\mathbf{A}}_{y}^{\prime}=\mathbf{A}_{y}^{\prime} \Pi \Delta_{3}+\mathbf{E}_{3},
\end{gathered}
$$

where $\Pi$ is a permutation matrix, and $\Delta_{1}, \Delta_{2}, \Delta_{3}$ note for the diagonal scaling matrices satisfying $\Delta_{1} \Delta_{2} \Delta_{3}=\mathbf{I}_{K} \cdot \mathbf{E}_{1}$, $\mathbf{E}_{2}$, and $\mathbf{E}_{3}$ are estimation error matrices. After the trilinear decomposition, the estimates of $\mathbf{A}_{x}^{\prime}, \mathbf{A}_{y}^{\prime}$, and $\mathbf{S}^{\prime}$ can be obtained. Scale ambiguity and permutation ambiguity are inherent to the trilinear decomposition problem. However, the scale ambiguity can be resolved easily by means of normalization, while the existence of permutation ambiguity is not considered for angle estimation.

3.3. Angle Estimation via Sparse Recovery. Use $\widehat{\mathbf{a}}_{x k}^{\prime}$ and $\widehat{\mathbf{a}}_{y k}^{\prime}$ to denote the $k$ th column of estimates $\widehat{\mathbf{A}}_{x}^{\prime}$ and $\widehat{\mathbf{A}}_{y}^{\prime}$, respectively. According to the compression matrices, we have

$$
\begin{aligned}
& \widehat{\mathbf{a}}_{x k}^{\prime}=\mathbf{U}^{T} \partial_{x k} \mathbf{a}_{x k}+\mathbf{n}_{x k}, \quad k=1, \ldots, K, \\
& \widehat{\mathbf{a}}_{y k}^{\prime}=\mathbf{V}^{T} \partial_{y k} \mathbf{a}_{y k}+\mathbf{n}_{y k}, \quad k=1, \ldots, K,
\end{aligned}
$$

where $\mathbf{a}_{x k}$ and $\mathbf{a}_{y k}$ are the $k$ th column of $\mathbf{A}_{x}, \mathbf{A}_{y}$, respectively. $\mathbf{n}_{x k}$ and $\mathbf{n}_{y k}$ are the corresponding noise, respectively. $\partial_{x k}$ and $\partial_{x k}$ are the scaling coefficients. Construct two Vandermonde matrices $\mathbf{A}_{s x} \in \mathbf{C}^{M \times P}$ and $\mathbf{A}_{s y} \in \mathbf{C}^{N \times P}(P \gg M, P \gg N)$ composed of steering vectors corresponding to each potential source location as its columns:

$$
\begin{aligned}
& \mathbf{A}_{s x} \\
& =\left[\mathbf{a}_{s x 1}, \mathbf{a}_{s x 2} \ldots, \mathbf{a}_{s x P}\right] \\
& =\left[\begin{array}{cccc}
1 & 1 & \cdots & 1 \\
e^{j 2 \pi d \mathbf{g}(1) / \lambda} & e^{j 2 \pi d \mathbf{g}(2) / \lambda} & \vdots & e^{j 2 \pi d \mathbf{g}(P) / \lambda} \\
\vdots & \vdots & \vdots & \vdots \\
e^{j 2 \pi(M-1) d \mathbf{g}(1) / \lambda} & e^{j 2 \pi(M-1) d \mathbf{g}(2) / \lambda} & \cdots & e^{j 2 \pi(M-1) d \mathbf{g}(P) / \lambda}
\end{array}\right],
\end{aligned}
$$

$$
\begin{aligned}
& \mathbf{A}_{s y} \\
& =\left[\mathbf{a}_{s y 1}, \mathbf{a}_{s y 2} \ldots, \mathbf{a}_{s y P}\right] \\
& =\left[\begin{array}{cccc}
1 & 1 & \cdots & 1 \\
e^{j 2 \pi d \mathbf{g}(1) / \lambda} & e^{j 2 \pi d \mathbf{g}(2) / \lambda} & \vdots & e^{j 2 \pi d \mathbf{g}(P) / \lambda} \\
\vdots & \vdots & \vdots & \vdots \\
e^{j 2 \pi(N-1) d \mathbf{g}(1) / \lambda} & e^{j 2 \pi(N-1) d \mathbf{g}(2) / \lambda} & \cdots & e^{j 2 \pi(N-1) d \mathbf{g}(P) / \lambda}
\end{array}\right],
\end{aligned}
$$

where $\mathbf{g}$ is a sampling vector and its $p$ th elements is $\mathbf{g}(p)=$ $-1+2 p / P, p=1,2, \ldots, P$. The matrices $\mathbf{A}_{s x}$ and $\mathbf{A}_{s y}$ can be regarded as the completed dictionaries. Then (27a)-(27b) can be expressed as

$$
\begin{aligned}
& \widehat{\mathbf{a}}_{x k}^{\prime}=\mathbf{U}^{T} \mathbf{A}_{s x} \mathbf{x}_{s}+\mathbf{n}_{x k}, \quad k=1, \ldots, K, \\
& \widehat{\mathbf{a}}_{y k}^{\prime}=\mathbf{V}^{T} \mathbf{A}_{y x} \mathbf{y}_{s}+\mathbf{n}_{y k}, \quad k=1, \ldots, K
\end{aligned}
$$

where $\mathbf{x}_{s}$ and $\mathbf{y}_{s}$ are sparse. The estimates of $\mathbf{x}_{s}$ and $\mathbf{y}_{s}$ can be obtained via $l_{0}$-norm constraint:

$$
\begin{array}{ll}
\min & \left\|\widehat{\mathbf{a}}_{x k}^{\prime}-\mathbf{U}^{T} \mathbf{A}_{s x} \mathbf{x}_{s}\right\|_{2}^{2}, \\
\text { st. } & \left\|\mathbf{x}_{s}\right\|_{0}=1, \\
\min & \left\|\widehat{\mathbf{a}}_{y k}^{\prime}-\mathbf{V}^{T} \mathbf{A}_{s y} \mathbf{y}_{s}\right\|_{2}^{2}, \\
\text { st. } & \left\|\mathbf{y}_{s}\right\|_{0}=1,
\end{array}
$$

where $\|\cdot\|_{0}$ denotes the $l_{0}$-norm. $\left\|\mathbf{x}_{s}\right\|_{0}=1$; that is to say, there is only one nonzero element in the vector $\mathbf{x}_{s}$, similar to $\left\|\mathbf{y}_{s}\right\|_{0}=1$. We can use the OMP recovery method [26] to find the nonzero element in $\mathbf{x}_{s}$ or $\mathbf{y}_{s}$. The OMP algorithm tries to recover the signal by finding the strongest component in the measurement signal, removing it from the signal, and searching the dictionary again for the strongest atom that is presented in the residual signal [32]. We extract the index of the maximum modulus of elements in $\mathbf{x}_{s}$ and $\mathbf{y}_{s}$, respectively, noted as $p_{x}$ and $p_{y}$. According to the corresponding columns in $\mathbf{A}_{s x}$ and $\mathbf{A}_{s y}$, we obtain $\mathbf{g}\left(p_{x}\right)$ and $\mathbf{g}\left(p_{y}\right)$, which are estimates of $\sin \theta_{k} \cos \phi_{k}$ and $\sin \theta_{k} \sin \phi_{k}$. We define 
$r_{k}=\mathbf{g}\left(p_{x}\right)+j \mathbf{g}\left(p_{y}\right)$, and then the elevation angles and azimuth angles can be obtained via

$$
\begin{aligned}
& \widehat{\theta}_{k}=\sin ^{-1}\left(\operatorname{abs}\left(r_{k}\right)\right), \quad k=1, \ldots, K, \\
& \widehat{\phi}_{k}=\operatorname{angle}\left(r_{k}\right), \quad k=1, \ldots, K,
\end{aligned}
$$

where $\operatorname{abs}(\cdot)$ is the modulus value symbol and angle $(\cdot)$ is to get the angle of an imaginary number. As the columns of the estimated matrices $\widehat{\mathbf{A}}_{x}^{\prime}$ and $\widehat{\mathbf{A}}_{y}^{\prime}$ are automatically paired, then the estimated elevation angles and azimuth angles can be paired automatically.

3.4. The Procedures of the Proposed Algorithm. Till now, we have achieved the proposal for the compressive sensing trilinear model-based 2D-DOA estimation for rectangular array. We show major steps of the proposed algorithm as follows.

Step 1. Form the three-way matrix $\overline{\mathbf{X}} \in \mathbf{C}^{M \times N \times L}$, then compress the three-way matrix into a much smaller threeway matrix $\overline{\mathbf{X}}^{\prime} \in \mathbf{C}^{M^{\prime} \times N^{\prime} \times L^{\prime}}$ via the compression matrices $\mathbf{U} \in \mathbf{C}^{M \times M^{\prime}}, \mathbf{V} \in \mathbf{C}^{N \times N^{\prime}}$, and $\mathbf{W} \in \mathbf{C}^{L \times L^{\prime}}$.

Step 2. Perform trilinear decomposition through TALS algorithm for the compressed three-way matrix to obtain the estimation of $\mathbf{A}_{x}^{\prime}, \mathbf{A}_{y}^{\prime}$, and $\mathbf{S}^{\prime}$.

Step 3. Estimate the sparse vectors.

Step 4. Estimate 2D-DOA via (31a)-(31b).

Remark A. Because the trilinear decomposition brings the same permutation ambiguity for the estimates $\mathbf{A}_{x}^{\prime}, \mathbf{A}_{y}^{\prime}$, and $\mathbf{S}^{\prime}$, the estimated elevation angles and azimuth angles are paired automatically.

Remark $B$. The conventional compressive sensing method formulates an angle sampling grid for sparse recovery to estimate angles. When it is applied to 2D-DOA estimation, both elevation and azimuth angles must be sampled, and it results in a two-dimensional sampling problem which brings much heavier cost for sparse signal recovery. In this paper, $\sin \theta_{k} \cos \phi_{k}$ (or $\sin \theta_{k} \sin \phi_{k}$ ) is bundled into a single variable in the range of -1 to 1 . The bundled variable is sampled for sparse recovery to obtain the estimates of $\sin \theta_{k} \cos \phi_{k}$ and $\sin \theta_{k} \sin \phi_{k}$, respectively. Afterwards, the elevation and azimuth angles are estimated through the estimates of $\sin \theta_{k} \cos \phi_{k}$ and $\sin \theta_{k} \sin \phi_{k}$.

Remark $C$. If the number of sources $K$ is unknown, it can be estimated by performing singular value decomposition for received data matrix $\mathbf{X}$ in (7) and finding the number of largest singular values [33]. We also use some lowercomplexity algorithm in [34] for estimating the number of the sources.

Remark $D$. When the coherent sources impinge on the array, we can use the parallel profiles with linear dependencies
(PARALIND) model $[35,36]$, which is a generalization of PARAFAC suitable for solving problems with linear dependent factors, to resolve coherent DOA estimation problem.

3.5. Complexity Analysis and CRB. The proposed algorithm has much lower computational cost than conventional trilinear decomposition-based method. The proposed algorithm requires $O\left(K^{3}+M^{\prime} N^{\prime} L^{\prime} K\right)$ operations for a iteration, while the trilinear decomposition algorithm needs $O\left(K^{3}+M N L K\right)$ operations [28] for a iteration, where $M^{\prime}<M, N^{\prime}<N$, and $L^{\prime}<L$.

We define $\mathbf{A}=\left[\mathbf{a}_{y}\left(\theta_{1}\right) \otimes \mathbf{a}_{x}\left(\theta_{1}\right), \ldots, \mathbf{a}_{y}\left(\theta_{K}\right) \otimes \mathbf{a}_{x}\left(\theta_{K}\right)\right]$. According to [37], we can derive the CRB

$$
\mathrm{CRB}=\frac{\sigma^{2}}{2 L}\left\{\operatorname{Re}\left[\mathbf{D}^{H} \boldsymbol{\Pi}_{\mathbf{A}}^{\perp} \mathbf{D} \oplus \widehat{\mathbf{P}}_{s}^{T}\right]\right\}^{-1},
$$

where $L$ denotes the number of samples, $\mathbf{a}_{k}$ is the $k$ th column of $\mathbf{A}$, and $\widehat{\mathbf{P}}_{s}=(1 / L) \sum_{t=1}^{L} \mathbf{s}(t) \mathbf{s}^{H}(t) . \sigma^{2}$ is the noise power. $\boldsymbol{\Pi}_{\mathbf{A}}^{\perp}=\mathbf{I}_{M N}-\mathbf{A}\left(\mathbf{A}^{H} \mathbf{A}\right)^{-1} \mathbf{A}^{H}$ and

$$
\mathbf{D}=\left[\frac{\partial \mathbf{a}_{1}}{\partial \theta_{1}}, \frac{\partial \mathbf{a}_{2}}{\partial \theta_{2}}, \ldots, \frac{\partial \mathbf{a}_{K}}{\partial \theta_{K}}, \frac{\partial \mathbf{a}_{1}}{\partial \phi_{1}}, \frac{\partial \mathbf{a}_{2}}{\partial \phi_{2}}, \ldots, \frac{\partial \mathbf{a}_{K}}{\partial \phi_{K}}\right] .
$$

The advantages of the proposed algorithm can be presented as follows.

(1) The proposed algorithm can be regarded as a combination of trilinear model and compressive sensing theory, and it brings much lower computational complexity and much smaller demand for storage capacity.

(2) The proposed algorithm has better 2D-DOA estimation performance than ESPRIT algorithm and close angle estimation performance to TALS algorithm, which will be proved by Figures 6-7.

(3) The proposed algorithm can achieve paired elevation angles and azimuth angles automatically.

\section{Numerical Simulations}

In the following simulations, we assume that the numerical simulation results converge when the SSR $\leq 10^{-8} . M, N, L$, and $K$ denote the number of antennas in $x$-axis, number of antennas in $y$-axis, samples, and sources, respectively. And we compress the parameters $M, N, L$ to $M^{\prime}, N^{\prime}$, and $L^{\prime}$ (usually set $M=16, N=20, L=100$, and $M^{\prime}=N^{\prime}=$ $L^{\prime}=5$ in numerical simulations). $d=\lambda / 2$ is considered in the simulation. We present 1000 Monte Carlo simulations to assess the angle estimation performance of the proposed algorithm. Define root mean squared error (RMSE) as

$$
\mathrm{RMSE}=\frac{1}{K} \sum_{k=1}^{K} \sqrt{\frac{1}{1000} \sum_{l=1}^{1000}\left(\widehat{\phi}_{k, l}-\phi_{k}\right)^{2}+\left(\widehat{\theta}_{k, l}-\theta_{k}\right)^{2}},
$$

where $\phi_{k}$ and $\theta_{k}$ denote the perfect elevation angle and azimuth angle of $k$ th source, respectively. $\widehat{\phi}_{k, l}$ and $\widehat{\theta}_{k, l}$ are 


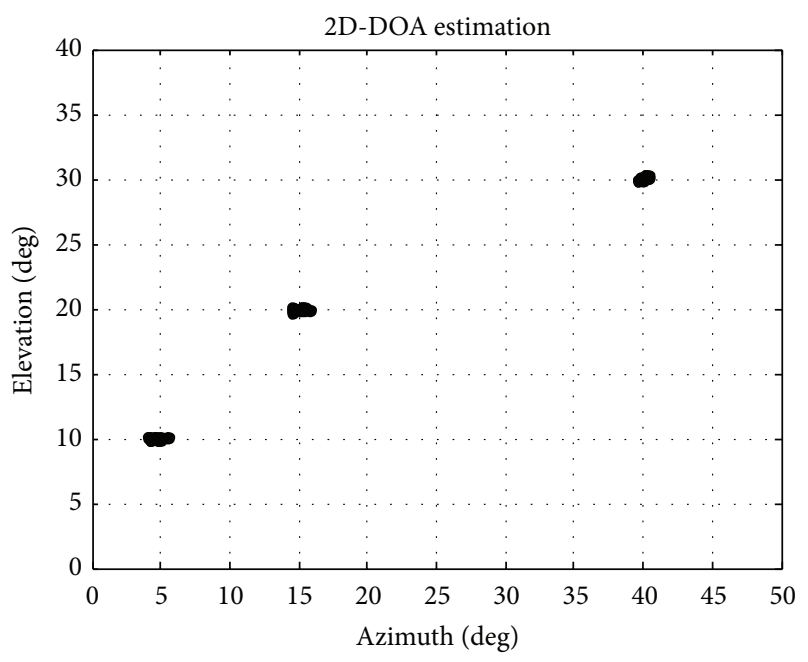

FIGURE 4: 2D-DOA estimation of our algorithm in SNR $=-10 \mathrm{~dB}$ $(N=20, M=16, L=100$, and $K=3)$.

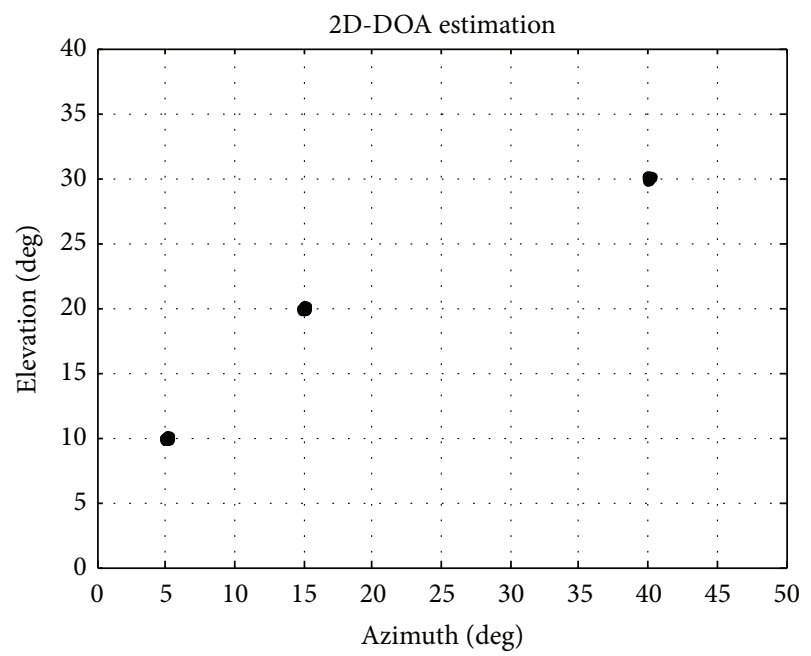

FIGURE 5: 2D-DOA estimation of our algorithm in SNR $=5 \mathrm{~dB}(N=$ $20, M=16, L=100$, and $K=3$ ).

the estimates of $\phi_{k}$ and $\theta_{k}$ in the $l$ th Monte Carlo trail. Assume that there are 3 noncoherent sources located at angles $\left(\phi_{1}, \theta_{1}\right)=\left(5^{\circ}, 10^{\circ}\right),\left(\phi_{2}, \theta_{2}\right)=\left(15^{\circ}, 20^{\circ}\right)$, and $\left(\phi_{3}, \theta_{3}\right)=$ $\left(40^{\circ}, 30^{\circ}\right)$.

Figure 4 presents the 2D-DOA estimation of the proposed algorithm for uniform rectangular array with $N=20$, $M=16, L=100, K=3$, and SNR $=-10 \mathrm{~dB}$. Figure 5 depicts the 2D-DOA estimation performance with SNR $=5 \mathrm{~dB}$. Figures 4-5 illustrate that our algorithm is effective for 2DDOA estimation.

Figure 6 shows the 2D-DOA estimation performance comparison of the proposed algorithm, the ESPRIT algorithm, the TALS algorithm, and the CRB for the uniform rectangular array with $N=20, M=16, L=100$, and $K=3$, while Figure 7 depicts the 2D-DOA estimation performance comparison with $N=16, M=16, L=200$, and $K=3$. It is indicated that our algorithm has better angle estimation

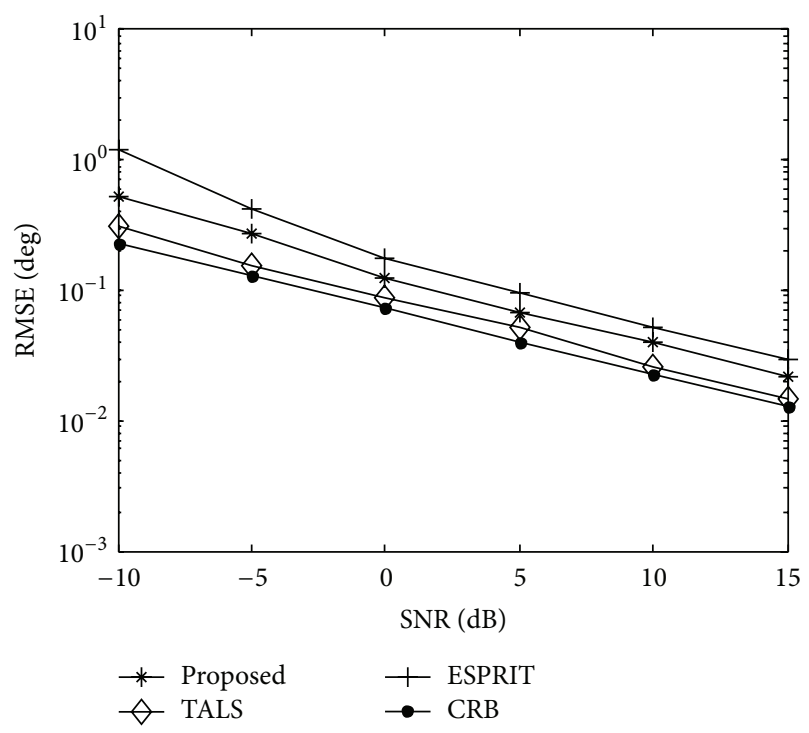

Figure 6: 2D-DOA estimation performance comparison $(N=20$, $M=16, L=100$, and $K=3$ ).

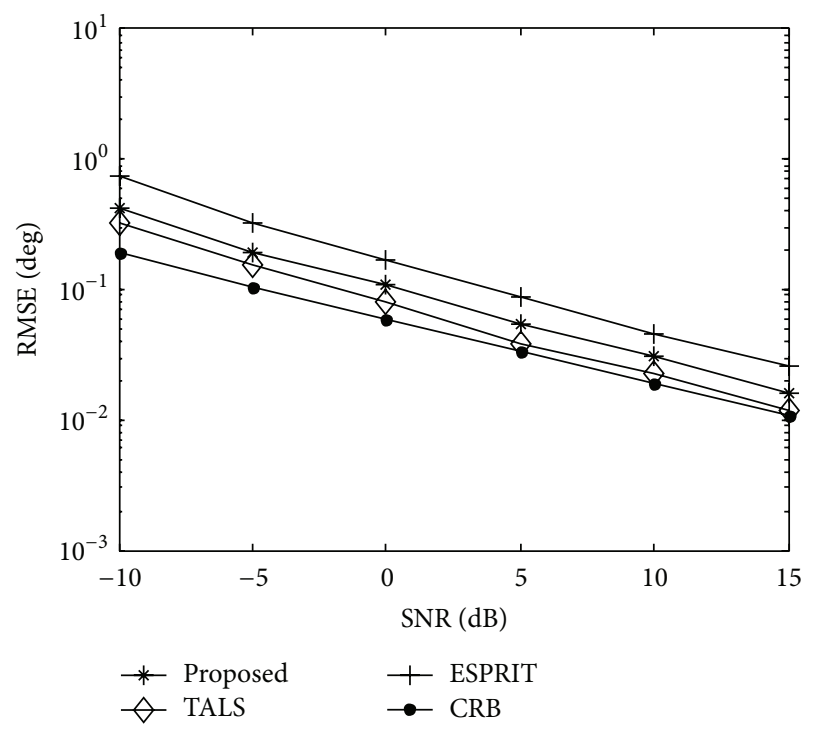

FigURE 7: 2D-DOA estimation performance comparison $(N=16$, $M=16, L=200$, and $K=3$ ).

performance than the ESPRIT algorithm and close angle estimation to TALS algorithm. The angle estimation performance of the proposed algorithm will be further improved through increasing the compressed parameters $M^{\prime}, N^{\prime}$, and $L^{\prime}$.

Figure 8 depicts the 2D-DOA estimation performance of the proposed algorithm with different value of $N(M=$ $16, L=100$, and $K=3$ ), while Figure 9 presents the 2D-DOA estimation performance of the proposed algorithm with different value of $M$. It is clearly shown that the angle estimation performance of our algorithm is gradually improved with the number of antennas increasing. Multiple 


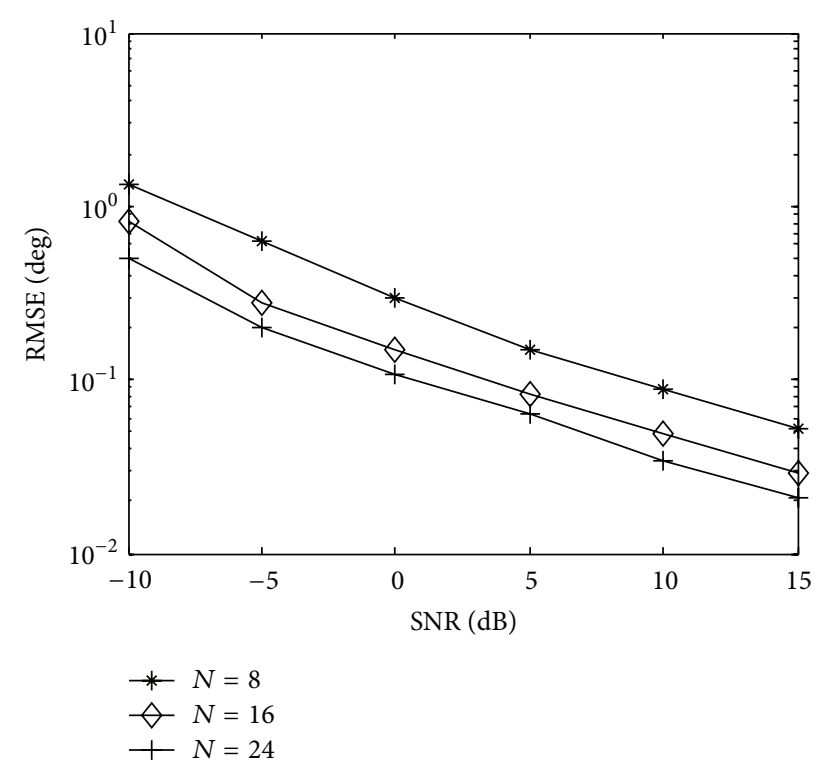

FIGURE 8: Angle estimation performance of our algorithm with different $N(M=16, L=100$, and $K=3)$.

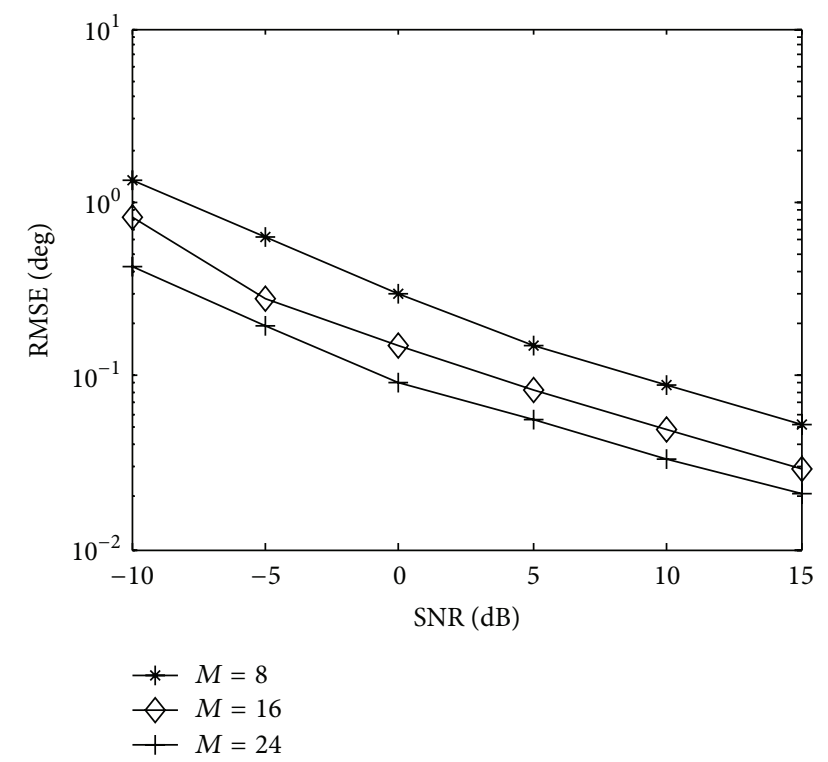

FIgURE 9: Angle estimation performance of our algorithm with different $M(N=16, L=100$, and $K=3)$.

antennas improve the angle estimation performance because of diversity gain.

Figure 10 presents 2D-DOA estimation performance of the proposed algorithm with different value of $L(N=$ 20, $M=16$, and $K=3$ ). It illustrates that the angle estimation performance becomes better in collaboration with $L$ increasing.

\section{Conclusions}

In this paper, we have addressed the 2D-DOA estimation problem for rectangular array and have derived a compressive

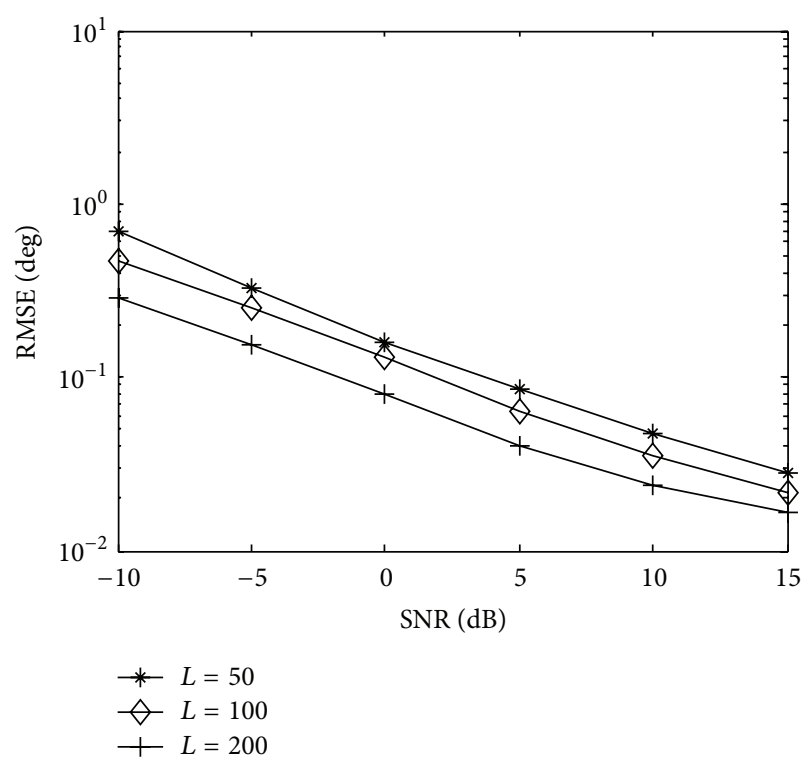

FIGURE 10: Angle estimation performance of our algorithm with different $L(N=16, M=20$, and $K=3)$.

sensing trilinear model-based 2D-DOA estimation algorithm, which can obtain the automatically paired 2D-DOA estimate. The proposed algorithm has better angle estimation performance than ESPRIT algorithm and close angle estimation performance to conventional trilinear decomposition method. Furthermore, the proposed algorithm has lower computational complexity and smaller demand for storage capacity than conventional trilinear decomposition method. The proposed algorithm can be regarded as a combination of trilinear model and compressive sensing theory, and it brings much lower computational complexity and much smaller demand for storage capacity.

\section{Conflict of Interests}

The authors declare that there is no conflict of interests regarding the publication of this paper.

\section{Acknowledgments}

This work is supported by China NSF Grants (61371169, 61301108, 61471191, 61471192, and 61271327), Jiangsu Planned Projects for Postdoctoral Research Funds (1201039C), China Postdoctoral Science Foundation (2012M521099, 2013M541661), Open Project of Key Laboratory of Modern Acoustics of Ministry of Education (Nanjing University), the Aeronautical Science Foundation of China (20120152001), Qing Lan Project, Priority Academic Program Development of Jiangsu High Education Institutions, and the Fundamental Research Funds for the Central Universities (NS2013024, kfjj130114, and kfjj130115). 


\section{References}

[1] H. Krim and M. Viberg, "Two decades of array signal processing research: the parametric approach," IEEE Signal Processing Magazine, vol. 13, no. 4, pp. 67-94, 1996.

[2] R. Roy and T. Kailath, "ESPRIT-estimation of signal parameters via rotational invariance techniques," IEEE Transactions on Acoustics, Speech and Signal Processing, vol. 37, no. 7, pp. 984995, 1989.

[3] F. Gao and A. B. Gershman, "A generalized ESPRIT approach to direction-of-arrival estimation," IEEE Signal Processing Letters, vol. 12, no. 3, pp. 254-257, 2005.

[4] D. Kundu, "Modified MUSIC algorithm for estimating DOA of signals," Signal Processing, vol. 48, no. 1, pp. 85-90, 1996.

[5] B. D. Rao and K. V. S. Hari, "Performance analysis of RootMusic," IEEE Transactions on Acoustics, Speech, and Signal Processing, vol. 37, no. 12, pp. 1939-1949, 1989.

[6] N. Yilmazer, J. Koh, and T. K. Sarkar, "Utilization of a unitary transform for efficient computation in the matrix pencil method to find the direction of arrival," IEEE Transactions on Antennas and Propagation, vol. 54, no. 1, pp. 175-181, 2006.

[7] Y. Chiba, K. Ichige, and H. Arai, "Reducing DOA estimation error in extended ES-root-MUSIC for uniform rectangular array," in Proceedings of the 4th International Congress on Image and Signal Processing (CISP '11), vol. 5, pp. 2621-2625, October 2011.

[8] T. Filik and T. E. Tuncer, "2-D paired direction-of-arrival angle estimation with two parallel uniform linear arrays," International Journal of Innovative Computing, Information and Control, vol. 7, no. 6, pp. 3269-3279, 2011.

[9] Y.-Y. Wang and S.-C. Huang, "An ESPRIT-based algorithm for 2D-DOA estimation," IEICE Transactions on Fundamentals of Electronics, Communications and Computer Sciences, vol. E94.A, no. 9, pp. 1847-1850, 2011.

[10] C. P. Mathews, M. Haardt, and M. D. Zoltowski, "Performance analysis of closed-form, ESPRIT based 2-D angle estimator for rectangular arrays," IEEE Signal Processing Letters, vol. 3, no. 4, pp. 124-126, 1996.

[11] M. D. Zoltowski, M. Haardt, and C. P. Mathews, "Closed-form 2-D angle estimation with rectangular arrays in element space or beamspace via unitary ESPRIT,' IEEE Transactions on Signal Processing, vol. 44, no. 2, pp. 316-328, 1996.

[12] N. D. Sidiropoulos, R. Bro, and G. B. Giannakis, "Parallel factor analysis in sensor array processing," IEEE Transactions on Signal Processing, vol. 48, no. 8, pp. 2377-2388, 2000.

[13] Y. Hua, "A pencil-MUSIC algorithm for finding twodimensional angles and polarizations using crossed dipoles," IEEE Transactions on Antennas and Propagation, vol. 41, no. 3, pp. 370-376, 1993.

[14] D. L. Donoho, "Compressed sensing," IEEE Transactions on Information Theory, vol. 52, no. 4, pp. 1289-1306, 2006.

[15] E. J. Candes, J. Romberg, and T. Tao, "Robust uncertainty principles: exact signal reconstruction from highly incomplete frequency information," IEEE Transactions on Information Theory, vol. 52, no. 2, pp. 489-509, 2006.

[16] D. Malioutov, M. Cetin, and A. S. Willsky, "A sparse signal reconstruction perspective for source localization with sensor arrays," IEEE Transactions on Signal Processing, vol. 53, no. 8, pp. 3010-3022, 2005.

[17] N. Hu, Z. Ye, D. Xu, and S. Cao, "A sparse recovery algorithm for DOA estimation using weighted subspace fitting," Signal Processing, vol. 92, no. 10, pp. 2566-2570, 2012.
[18] J. B. Kruskal, “Three-way arrays: rank and uniqueness of trilinear decompositions, with application to arithmetic complexity and statistics," Linear Algebra and its Applications, vol. 18, no. 2, pp. 95-138, 1977.

[19] L. de Lathauwer, B. de Moor, and J. Vandewalle, "Computation of the canonical decomposition by means of a simultaneous generalized Schur decomposition," SIAM Journal on Matrix Analysis and Applications, vol. 26, no. 2, pp. 295-327, 2004.

[20] L. de Lathauwer, "A link between the canonical decomposition in multi-linear algebra and simultaneous matrix diagonalization," SIAM Journal on Matrix Analysis and Applications, vol. 28, no. 3, pp. 642-666, 2006.

[21] N. D. Sidiropoulos, G. B. Giannakis, and R. Bro, "Blind PARAFAC receivers for DS-CDMA systems," IEEE Transactions on Signal Processing, vol. 48, no. 3, pp. 810-823, 2000.

[22] N. D. Sidiropoulos and X. Liu, "Identifiability results for blind beamforming in incoherent multipath with small delay spread," IEEE Transactions on Signal Processing, vol. 49, no. 1, pp. 228236, 2001.

[23] N. D. Sidiropoulos and A. Kyrillidis, "Multi-way compressed sensing for sparse low-rank tensors," IEEE Signal Processing Letters, vol. 19, no. 11, pp. 757-760, 2012.

[24] X. F. Zhang, H. X. Yu, J. F. Li, and D. Ben, "Blind signal detection for uniform rectangular array via compressive sensing trilinear model," Advanced Materials Research, vol. 756-759, pp. 660664, 2013.

[25] R. Cao, X. Zhang, and W. Chen, "Compressed sensing parallel factor analysis-based joint angle and Doppler frequency estimation for monostatic multiple-input-multiple-output radar," IET Radar, Sonar \& Navigation, vol. 8, no. 6, pp. 597-606, 2014.

[26] J. A. Tropp and A. C. Gilbert, "Signal recovery from random measurements via orthogonal matching pursuit," IEEE Transactions on Information Theory, vol. 53, no. 12, pp. 4655-4666, 2007.

[27] X. Zhang, F. Wang, and H. Chen, Theory and Application of Array Signal Processing (version 2), National Defense Industry Press, Beijing, China, 2012.

[28] X. Zhang, J. Li, H. Chen, and D. Xu, "Trilinear decompositionbased two-dimensional DOA estimation algorithm for arbitrarily spaced acoustic vector-sensor array subjected to unknown locations," Wireless Personal Communications, vol. 67, no. 4, pp. 859-877, 2012.

[29] R. Bro, N. D. Sidiropoulos, and G. B. Giannakis, "A fast least squares algorithm for separating trilinear mixtures," in Proceedings of the International Workshop on Independent Component Analysis and Blind Signal Separation, pp. 289-294, January 1999.

[30] R. A. DeVore, "Deterministic constructions of compressed sensing matrices," Journal of Complexity, vol. 23, no. 4-6, pp. 918-925, 2007.

[31] S. Li and X. Zhang, "Study on the compressed matrices in compressed sensing trilinear model," Applied Mechanics and Materials, vol. 556-562, pp. 3380-3383, 2014.

[32] F. Wang and X. Zhang, "Joint estimation of TOA and DOA in IR-UWB system using sparse representation framework," ETRI Journal, vol. 36, no. 3, pp. 460-468, 2014.

[33] A. Di, "Multiple source location-a matrix decomposition approach," IEEE Transactions on Acoustics, Speech, and Signal Processing, vol. 33, no. 5, pp. 1086-1091, 1985.

[34] J. Xin, N. Zheng, and A. Sano, "Simple and efficient nonparametric method for estimating the number of signals without eigendecomposition," IEEE Transactions on Signal Processing, vol. 55, no. 4, pp. 1405-1420, 2007. 
[35] R. Bro, R. A. Harshman, N. D. Sidiropoulos, and M. E. Lundy, "Modeling multi-way data with linearly dependent loadings," Journal of Chemometrics, vol. 23, no. 7-8, pp. 324-340, 2009.

[36] X. Zhang, M. Zhou, and J. Li, "A PARALIND decompositionbased coherent two-dimensional direction of arrival estimation algorithm for acoustic vector-sensor arrays," Sensors, vol. 13, no. 4, pp. 5302-5316, 2013.

[37] P. Stoica and A. Nehorai, "Performance study of conditional and unconditional direction-of-arrival estimation," IEEE Transactions on Acoustics, Speech, and Signal Processing, vol. 38, no. 10, pp. 1783-1795, 1990. 

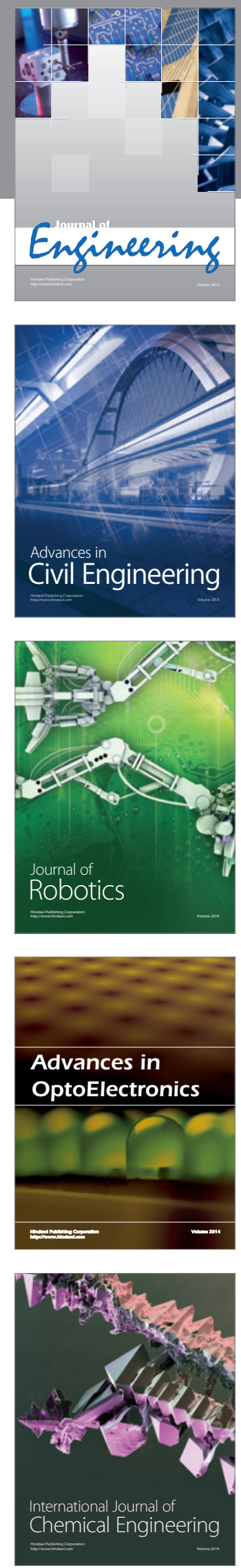

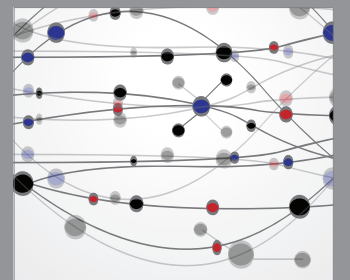

The Scientific World Journal
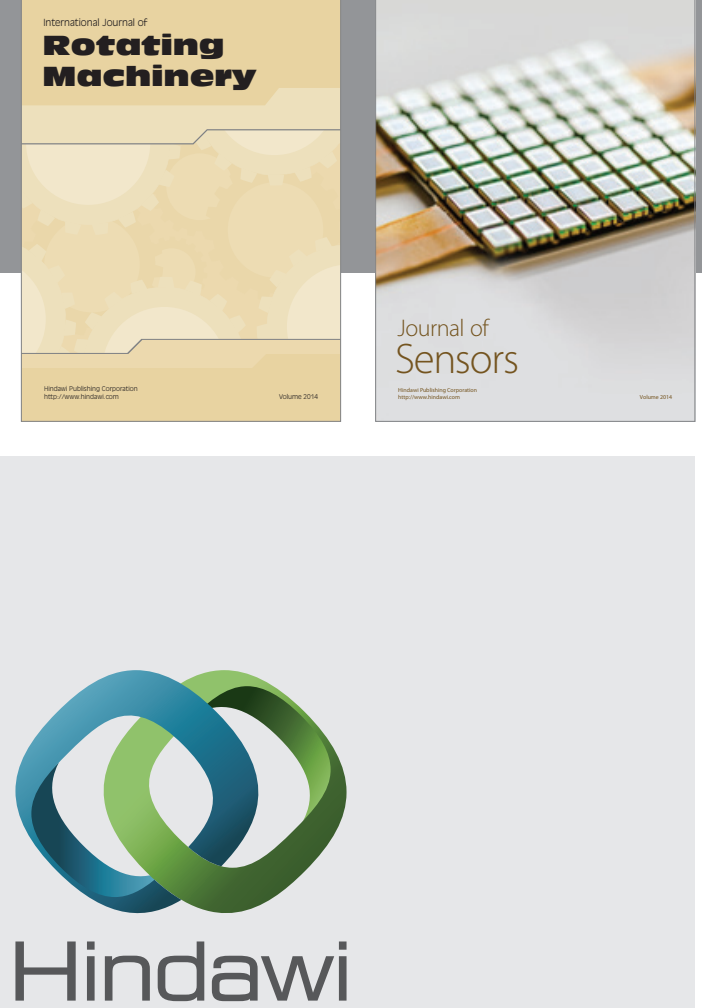

Submit your manuscripts at http://www.hindawi.com
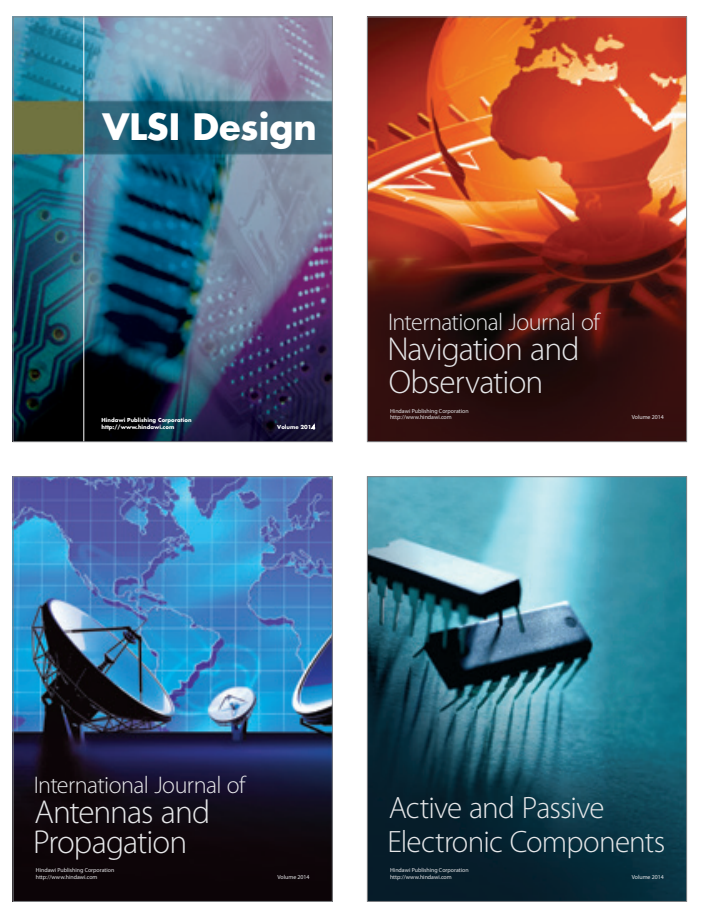
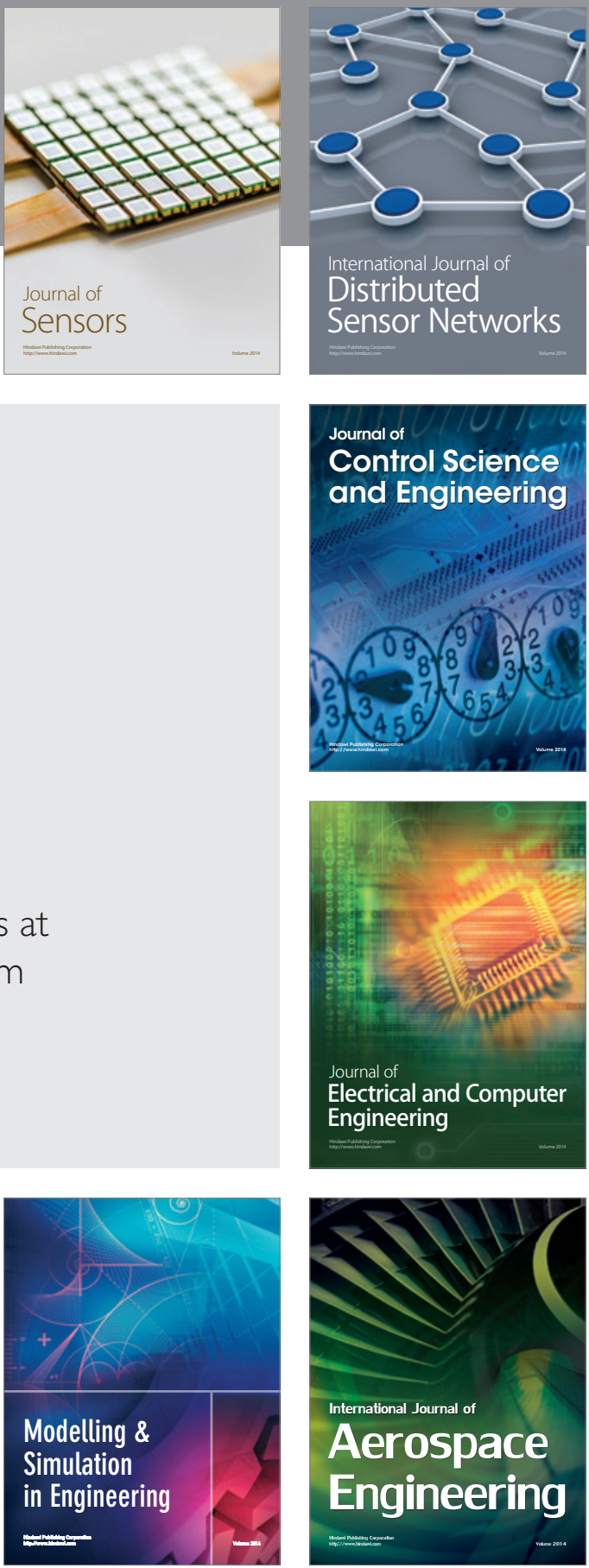

Journal of

Control Science

and Engineering
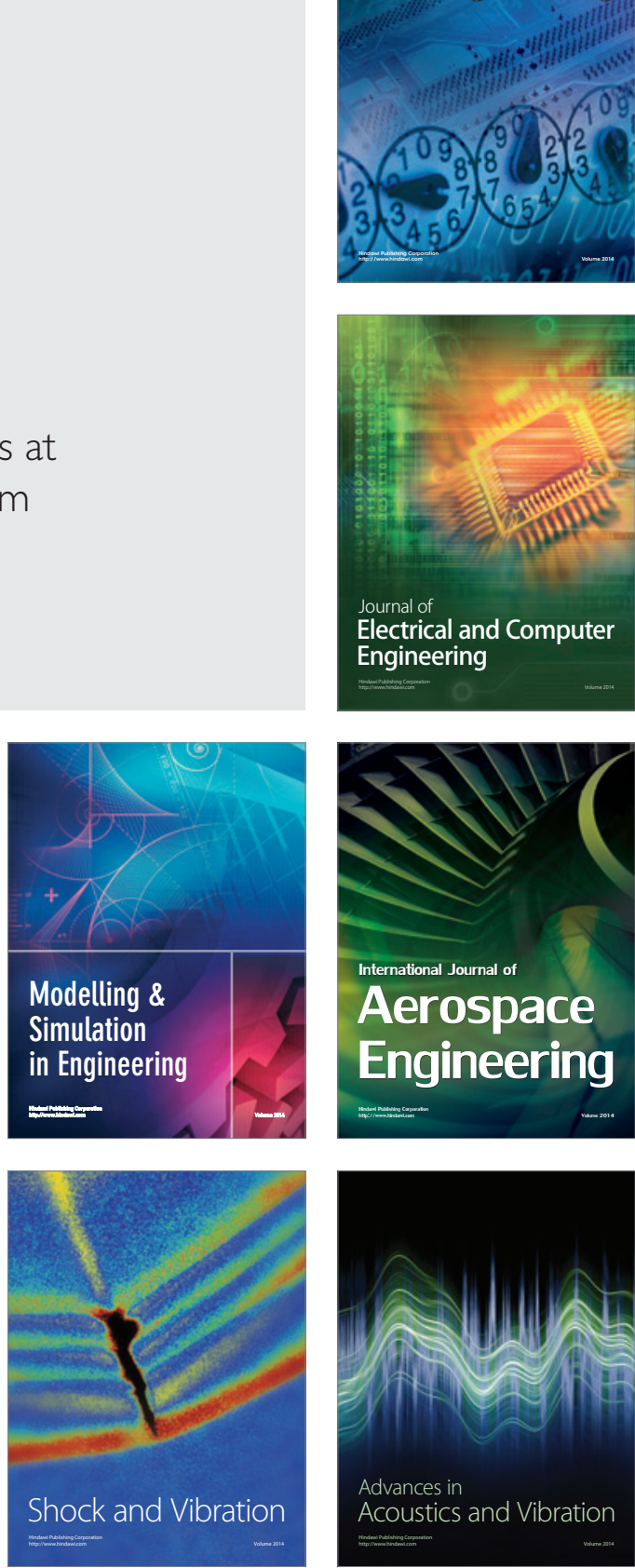